\title{
ON A NOTION OF SMALLNESS FOR SUBSETS OF THE BAIRE SPACE
}

BY

\author{
ALEXANDER S. KECHRIS
}

\begin{abstract}
Let us call a set $A \subseteq \omega^{\omega}$ of functions from $\omega$ into $\omega \sigma$-bounded if there is a countable sequence of functions $\left\{\alpha_{n}: n \in \omega\right\} \subseteq \omega^{\omega}$ such that every member of $A$ is pointwise dominated by an element of that sequence. We study in this paper definability questions concerning this notion of smallness for subsets of $\omega^{\omega}$. We show that most of the usual definability results about the structure of countable subsets of $\omega^{\omega}$ have corresponding versions which hold about $\sigma$-bounded subsets of $\omega^{\omega}$. For example, we show that every $\Sigma_{2 n+1}^{1} \sigma$-bounded subset of $\omega^{\omega}$ has a $\Delta_{2 n+1}^{1}$ "bound" $\left\{\alpha_{m}: m \in \omega\right\}$ and also that for any $n \geqslant 0$ there are largest $\sigma$-bounded $\Pi_{2 n+1}^{1}$ and $\Sigma_{2 n+2}^{1}$ sets. We need here the axiom of projective determinacy if $n \geqslant 1$. In order to study the notion of $\sigma$-boundedness a simple game is devised which plays here a role similar to that of the standard *-games (see [My]) in the theory of countable sets. In the last part of the paper a class of games is defined which generalizes the *-and ${ }^{* *}$ - (or Banach-Mazur) games (see [My]) as well as the game mentioned above. Each of these games defines naturally a notion of smallness for subsets of $\omega^{\omega}$ whose special cases include countability, being of the first category and $\sigma$-boundedness and for which one can generalize all the main results of the present paper.
\end{abstract}

1. Preliminaries. 1A. Let $\omega=\{0,1,2, \ldots\}$ be the set of all natural numbers and $\Re=\omega^{\omega}$ the set of all functions from $\omega$ to $\omega$ or, for simplicity, reals. Letters $i, j, k, l, m, n, \ldots$ denote elements of $\omega$ and $\alpha, \beta, \gamma, \delta, \ldots$ reals. We study subsets of the product spaces $\mathcal{X}=X_{1} \times X_{2} \times \cdots \times X_{k}$, where $X_{i}$ is $\omega$ or $\Re_{\text {. We }}$ call such subsets pointsets. Sometimes we think of them as relations and we write interchangeably $x \in A \Leftrightarrow A(x)$. A pointclass is a class of pointsets, usually in all product spaces. We shall be concerned primarily in this paper with the analytical pointclasses $\Sigma_{n}^{1}, \Pi_{n}^{1}, \Delta_{n}^{1}$ and their corresponding projective pointclasses $\Sigma_{n}^{1}, \Pi_{n}^{1}, \Delta_{n}^{1}$. For information about them we refer the reader to $[\mathbf{R}]$, $[\mathbf{S h}]$ and $\left[\mathbf{M o}_{1}\right]$.

If $\Gamma$ is a pointclass and $\mathscr{X}$ a product space $(\mathcal{X}=\omega$ or $\mathcal{X}=\mathscr{R}$ will be enough for this definition), then we say that $\Gamma$ is $\mathcal{X}$-parametrized if for any product space $\mathcal{Y}$ there is a $G \in \Gamma, G \subseteq \mathcal{X} \times \mathscr{Y}$ such that letting $G_{x}=\{y:(x, y) \in G\}$ we have $\{A \subseteq \mathcal{Y}: A \in \Gamma\}=\left\{G_{x}: x \in X\right\}$. In this case $G$ is called $X$-universal

Received by the editors December 10, 1975.

AMS (MOS) subject classifications (1970). Primary 04A15, 02K30, 28A05, 54H05; Secondary 02F $35,02 \mathrm{~K} 05,02 \mathrm{~K} 25,02 \mathrm{~K} 35,04 \mathrm{~A} 30$. 
for $\Gamma$ subsets of $\mathscr{Y}$. If $A=G_{x}$ we call $x$ a code of $A$. It is well known that $\Sigma_{n}^{1}, \Pi_{n}^{1}$ are $\omega$-parametrized and $\Sigma_{n}^{1}, \Pi_{n}^{1}$ are $\mathcal{R}_{\text {-parametrized with universal sets }}$ which are actually in $\Sigma_{n}^{1}, \Pi_{n}^{1}$ respectively.

1B. The notions of games and determinacy are used repeatedly in this paper. For information about them which will be used without explicit reference the reader could consult $[\mathrm{My}],\left[\mathrm{Mo}_{2}\right],[\mathrm{Fe}]$ or $\left[\mathrm{Mo}_{1}\right]$. For any pointclass $\Gamma$,

\section{Determinacy $(\Gamma)$}

abbreviates the statement: Every $A \subseteq \Re, A \in \Gamma$ is determined. We also abbreviate

$$
\mathrm{PD} \Leftrightarrow \text { every projective set of reals is determined }
$$

and

$$
\mathrm{AD} \Leftrightarrow \text { every set of reals is determined. }
$$

1C. We shall frequently talk about trees. Given a set $X$, a tree on $X$ is a set of finite sequences from $X$, closed under subsequences i.e.

$$
\left(x_{0}, \ldots, x_{n}\right) \in T \& k \leqslant n \Rightarrow\left(x_{0} \cdots x_{k}\right) \in T .
$$

The empty sequence is always a member of a nonempty tree. A node of a tree is just a sequence in that tree. A branch of a tree $T$ is an infinite sequence $f \in X^{\omega}$ such that for every $n,(f(0), \ldots, f(n)) \in T$. The set of all branches of $T$ is denoted by

$$
[T]=\left\{f \in X^{\omega}: \forall n(f(0) \cdots f(n)) \in T\right\} .
$$

We shall denote by $u \prec v$ the relation of proper extension between finite sequences. Thus if $u=\left(x_{0} \cdots x_{n}\right), v=\left(y_{0} \cdots y_{m}\right)$, then

$$
u \prec v \Leftrightarrow n>m \& \forall i \leqslant m\left(x_{i}=y_{i}\right) \text {. }
$$

We shall also denote by $u v$ the concatenation of two finite sequences $u, v$. Thus if $u=\left(x_{0} \cdots x_{n}\right), v=\left(y_{0} \cdots y_{m}\right)$, then

$$
u v=\left(x_{0} \cdots x_{n} y_{0} \cdots y_{m}\right) \text {. }
$$

If $u_{0}, u_{1}, u_{2}, \ldots$ is an infinite list of finite sequences we shall also denote by $u_{0}^{-} u_{1}^{-} u_{2}^{-} \cdots$ their concatenation. Thus if $u_{0}=\left(x_{0}^{0} \cdots x_{m_{0}}^{0}\right), u_{1}=\left(x_{0}^{1} \cdots x_{m_{1}}^{1}\right)$, $\ldots$, then

$$
u_{0}{ }^{-} u_{1}^{-} u_{2}^{-} \cdots=\left(x_{0}^{0} x_{1}^{0} \cdots x_{m_{0}}^{0} x_{0}^{1} x_{1}^{1} \cdots x_{m_{1}}^{1} \cdots\right)
$$

(this in general will be an infinite sequence, unless all $u_{i}$ after a certain point are empty). 
Occasionally we shall have to deal with trees on sets $X=Y \times Z$. A tree $T$ on $Y \times Z$ contains elements of the form $\left(\left(y_{0}, z_{0}\right), \ldots,\left(y_{n}, z_{n}\right)\right)$, where $y_{i} \in Y$, $z_{i} \in Z$. A branch of such a tree is a sequence $f \in(Y \times Z)^{\omega}$ which for convenience will be represented by the unique pair $(g, h)$ such that $f(n)$ $=(g(n), h(n))$. The first projection of $[T]$, in symbols $p[T]$, is

$$
p[T]=\{g: \exists h(g, h) \in[T]\} .
$$

Most of the time we shall have $Y=\omega, Z=\lambda$ ( $\lambda$ some ordinal). Then $p[T] \subseteq \Re$.

1D. Let $X$ (an arbitrary set containing more than one element) have the discrete topology. We shall always think of $X^{\omega}=$ set of infinite sequences of elements of $X$ as having the product topology. Open sets in this topology are generated by the basic neighborhoods $N_{u}=\left\{f \in X^{\omega}: f\right.$ extends $\left.u\right\}$, where $u$ ranges over the finite sequences from $X$. One can also visualize easily the closed sets of the space $X^{\omega}$. Say that a tree $T$ on $X$ has no finite branches if for every $u \in T$ there is a proper extension $v \prec u$ in $T$. Then the map

$$
A \mapsto\{(f(0) \cdots f(n)): f \in A, n \in \omega\} \stackrel{\text { def }}{=} T_{A}
$$

gives a 1-1 correspondence between closed sets of $X^{\omega}$ and trees with no finite branches on $X$ such that $A=\left[T_{A}\right]$. It is easy to see that $A$ is perfect iff every $u \in T_{A}$ has at least two incompatible extensions in $T_{A}$ ( $u, v$ are incompatible iff they have no common extension). Also $A$ is compact iff $T_{A}$ is finite splitting i.e. every $u \in T_{A}$ has only finitely many immediate extensions $u(x)$ in $T_{A}$. In case $X=\omega$ note that a closed set $A=\left[T_{A}\right]$ is compact iff there is a real $\beta$ such that for every $\alpha \in A, \forall n(\alpha(n) \leqslant \beta(n))$.

REMARK. In case $X=\omega$ the product spaces will be also equipped with the product topology.

For each countable $X, X^{\omega}$ is a perfect Polish (i.e. completely metrizable and separable) space. Topological notions related to category will be used as a tool repeatedly in this paper. In particular the following result will be needed in several places. If one replaces its hypothesis by PD then the result can be already essentially attributed to Banach-Mazur and Oxtoby (see for example [O]). It is not clear who noticed first the present stronger version but the key idea of using "witnesses" in various standard games already occurs in early unpublished results of Solovay (see for example [Bu]). Martin [ $\left.\mathbf{M a}_{2}\right]$ has also used *-games (see [My]) with witnesses in order to prove that, under Determinacy $\left(\Delta_{2 n}^{1}\right)$, every $\Sigma_{2 n+1}^{1}$ set with no nonempty perfect subset contains only $\Delta_{2 n+1}^{1}$ reals.

Theorem (Folklore). Assume Determinacy $\left(\Delta_{2 n}^{1}\right), n \geqslant 0$. Let $\mathcal{X}$ be any product space. Then every $\Sigma_{2 n+1}^{1}$ (and thus $\Pi_{2 n+1}^{1}$ ) set in $\mathcal{X}$ has the property of 
Baire. Similarly assuming Determinacy $\left(\Sigma_{2 n+1}^{1}\right), n \geqslant 1$, every $\Sigma_{2 n+2}^{1}$ (and thus $\left.\Pi_{2 n+2}^{1}\right)$ set has the property of Baire.

Proof. We prove the first assertion. The second can be proved in exactly the same way. It is also enough to consider the case $\mathcal{X}=\Re$.

Assume now $A \subseteq \Re$ and $A \in \Sigma_{2 n+1}^{1}$. Let $B \in \Pi_{2 n}^{1}$ be such that $\alpha \in A$ $\Leftrightarrow \exists \beta B(\alpha, \beta)$. Consider the following modified Banach-Mazur game in which player I plays also witnesses, which we shall denote by $G_{p}^{* *}(B)$ (the **. notation for the Banach-Mazur game comes from [My]): Player I plays $k_{0} \in \omega$ and a finite sequence $s_{0}$ from $\omega$, II plays a nonempty finite sequence $s_{1}$ from $\omega$, I plays $k_{2} \in \omega$ and a nonempty finite sequence $s_{2}$ from $\omega$, II plays a nonempty finite sequence $s_{3}$ from $\omega$, etc. At the end of the run of the game let $\beta=\left(k_{0}, k_{1}, k_{2}, \ldots\right) \in \Re, \alpha=s_{0}{ }^{-} s_{1}{ }^{-} \cdots \in \Re$. Then I wins iff $(\alpha, \beta)$ $\in B$. Otherwise II wins. We have now the following

LEMMA. (i) If I has a winning strategy in $G_{p}^{* *}(B)$, then for some open nonempty set $G, A$ is comeager on $G$.

(ii) If II has a winning strategy in $G_{p}^{* *}(B)$, then $A$ is meager.

Proof. (i) If I has a winning strategy with first move $\left(k_{0}, s_{0}\right)$, then it is easy to see that $B$ is comeager of $N_{s_{0}}=\left\{\alpha: s_{0}\right.$ is an initial segment of $\left.\alpha\right\}$; see for example [0, p. 28].

(ii)(1) Assume II has a winning strategy $\tau$. Call a sequence $\left(k_{0}, s_{0}, s_{1}, \ldots\right.$, $\left.k_{2 k}, s_{2 k}, s_{2 k+1}\right)$ good if for all $i \leqslant k, s_{2 i+1}$ is played according to $\tau$. By convention the empty sequence is good. If $(\alpha, \beta) \in B$ then there must be some good sequence $u=\left(k_{0}, s_{0}, s_{1}, \ldots, k_{2 k}, s_{2 k}, s_{2 k+1}\right)$ (maybe empty) such that $\left(k_{0}, k_{2}, \ldots, k_{2 k}\right)=(\beta(0) \cdots \beta(k))$ is an initial segment of $\beta$ and $s_{0} s_{1} \cdots \cdots s_{2 k+1}$ is an initial segment of $\alpha$, but no good extension $\left(k_{0}, s_{0}, k_{1}, \ldots\right.$, $\left.k_{2 k}, s_{2 k}, s_{2 k+1}, k_{2 k+2}, s_{2 k+2}, s_{2 k+3}\right)$ of $u$ has this property (otherwise $(\alpha, \beta)$ could be conceived as a play of the game in which II plays according to his strategy $\tau)$. Let $\beta(k+1)=m$ and put $M_{u, m}=\left\{\alpha^{\prime}: s_{0}{ }^{-} s_{1} \cdots \cdots s_{2 k+1}\right.$ is an initial segment of $\alpha^{\prime}$ and for every $s_{2 k+2}$, if

$$
s_{2 k+3}=\tau\left(k_{0}, s_{0}, \ldots, k_{2 k+1}, s_{2 k+1}, m, s_{2 k+2}\right) \text {, }
$$

then $s_{0}^{\hat{s}} s_{1} \cdots s_{2 k+3}$ is not an initial segment of $\left.\alpha^{\prime}\right\}$. Then $M_{u, m}$ is closed with no interior, i.e. it is nowhere dense and $\alpha \in M_{u, m}$. Since there are only countably many $M_{u, m}$ 's, $A$ is meager.

From this lemma it follows that (granting Determinacy $\left(\Delta_{2 n}^{1}\right)$, which by a result of Martin $\left[\mathrm{Ma}_{1}\right]$ implies Determinacy $\left(\Sigma_{2 n}^{1}\right)$ ) every $\Sigma_{2 n+1}^{1}$ set is meager (thus has the property of Baire) or it is comeager on an open set. Given now $A \in \Sigma_{2 n+1}^{1}$ consider $G=\bigcup\left\{N_{s}: A\right.$ is comeager on $\left.N_{s}\right\}$ (where for $s$ a finite

(l) We would like to thank Sy Friedman for helping simplify considerably the presentation of this part of the proof. 
sequence from $\omega, N_{s}=\{\alpha: \alpha$ extends $s\}$ ). Then $A$ is comeager in $G$, thus $G-A$ is meager. But also $A-G$ is $\Sigma_{2 n+1}^{1}$ and $A-G$ is not comeager on any open set, therefore $A-G$ is meager. So $(A-G) \cup(G-A)=A \triangle G$ is meager, i.e. $A$ has the property of Baire.

REMARK. A similar result can be proved about Lebesgue measurability using the Mycielski-Swierczkowski game (see [My-S]) with witnesses, which is essentially the game Solovay used in his proof that $A D \Rightarrow B C$ (see [Bu]) or the Harrington game (see for example [Ke $\left.\mathbf{K}_{\mathbf{1}}\right]$ ) with witnesses.

1E. The whole discussion in this paper takes place in $\mathrm{ZF}+\mathrm{DC}$, ZermeloFraenkel set theory with dependent choices:

$$
\forall u \in x \exists v(u, v) \in r \Rightarrow \exists f \forall n(f(n), f(n+1)) \in r .
$$

Every additional hypothesis is stated explicitly.

Our set theoretic notation and terminology will be standard, when possible. We shall use letters $\xi, \eta, \theta, \lambda, \ldots$ to denote ordinals.

2. $\sigma$-bounded and superperfect sets. 2A. Let $A \subseteq \Re$. We call $A \sigma$-bounded iff there is a sequence $\left\{\alpha_{i}\right\}_{i \in \omega}$ of reals such that for each $\alpha \in A$ there is some $i \in \omega$ with $\alpha \leqslant \alpha_{i}$, where for any two reals $\alpha, \beta$

$$
\alpha \leqslant \beta \stackrel{\text { def }}{\Leftrightarrow} \forall n(\alpha(n) \leqslant \beta(n)) .
$$

We call $\left\{\alpha_{i}\right\}_{i \in \omega}$ a bound for $A$.

Note here that $A$ is $\sigma$-bounded iff $A$ is contained in a $\sigma$-compact (i.e. a countable union of compact sets) subset of $R$ iff there is a $\beta$ such that for all $\alpha \in A, \alpha \leqslant * \beta$, where for any two reals $\alpha, \beta$

$$
\alpha \leqslant * \stackrel{\text { def }}{\Leftrightarrow} \exists i \forall n \geqslant i(\alpha(n) \leqslant \beta(n)) .
$$

We think of course of $\sigma$-boundedness as a notion of smallness for sets of reals, analogous to countability. Opposite to countable stands the concept of a perfect set. We shall now define the corresponding concept which provides the opposite to $\sigma$-boundedness.

Definition. Let $T$ be a tree on $\omega$. We call $T$ superperfect iff for every $u \in T$ there is $v \in T$ extending $u$ such that $\left\{m \in \omega: v^{-}(m) \in T\right\}$ is infinite i.e. $v$ has infinitely many immediate extensions in $T$. We shall call a set $A \subseteq \Re$ superperfect iff $A$ is closed and the tree of $A, T_{A}$ is superperfect.

It is easy to see that $A \subseteq \Re$ is superperfect iff $A$ is closed and for every $\alpha \in A$ and any open set $G$ containing $\alpha, G \cap A$ is not contained in a compact set. Also notice that every nonempty superperfect set contains a nonempty superperfect set homeomorphic to $\Re$.

REMARKs. (1) The notion of a superperfect tree is already implicit in Friedman $\left[\mathbf{F r}_{\mathbf{1}}\right]$, which provided some of the original motivation for studying the notions under consideration here. Lemma 1.3 in $\left[\mathbf{F r}_{\mathbf{1}}\right]$ is in our terminology 
essentially the statement: Every nonempty $\Pi_{1}^{0}$ set which contains no $\Delta_{1}^{1}$ real contains a nonempty superperfect set.

(2) (The axiom of choice is used in this remark.) We call $A \subseteq R$-bounded, where $\kappa$ is an infinite cardinal, if there is a sequence $\left\{\beta_{\xi}\right\}_{\xi<\kappa}$ of reals such that for each $\alpha \in A$ there is a $\xi<\kappa$ with $\alpha \leqslant \beta_{\xi}$. Clearly $A$ is $\kappa$-bounded iff there is a $\kappa$-compact (i.e. the union of $\kappa$ many compact sets) subset of $R$ containing $A$. Put for any $A \subseteq \Re, \#_{A}=$ least $\kappa$ such $A$ is $\kappa$ bounded. Let also $\kappa_{0}=\#_{R}$. Clearly $\kappa_{0}>\kappa_{0}$. Moreover $\kappa_{0}=$ least $\kappa$ such that $\kappa$ is the cardinality of a scale on $\Re$, where $S \subseteq \Re$ is a scale if $S$ is cofinal with respect to $\leqslant^{*}$. Thus from results of Solovay (unpublished) and Hechler $[\mathbf{H}]$ it follows that the value of $\kappa_{0}$ is consistently with ZFC "essentially independent" of $2^{\kappa_{0}}$. For example one could have $\kappa_{0}=2^{\kappa_{0}}>\kappa_{1}$ or $\kappa_{0}=\kappa_{1}$ and $2^{\kappa_{0}}=\aleph_{17}$. Notice also that if $A \neq \varnothing$ is superperfect, then $\#_{A}=2^{\aleph_{0}}$, since $\Re$ is homeomorphic to a closed subset of $A$.

2B. One of our main purposes in this paper is to establish results about $\sigma$ bounded and superperfect sets which are analogous to those about countable and perfect sets. As a simple start let us notice for example that as usual the analog of the Cantor-Bendixson Theorem goes through.

Proposition. Let $A \subseteq \Re$ be a closed set. Then we can write $A$ uniquely as $A=P \cup C$, where $P$ is superperfect, $C$ is o-bounded and $P \cap C=\varnothing$. In particular a closed set is either $\sigma$-bounded or contains a nonempty superperfect set.

Proof. Let $A=[T]$, where $T$ is a tree on $\omega$. For any tree $J$ on $\omega$ define the derivative:

$$
J^{\prime}=\{s \in J: \exists t(t \prec s \& t \in J \&\{m: \hat{t}(m) \in J\} \text { is infinite })\} .
$$

Then let by induction: $T_{0}=T, T_{\xi+1}=\left(T_{\xi}\right)^{\prime}, T_{\lambda}=\bigcap_{\xi<\lambda} T_{\xi}$, if $\lambda$ is limit. If $\xi_{0}$ is the least ordinal $\xi$ for which $T_{\xi}=T_{\xi+1}$, then $\xi_{0}$ is countable and $P=\left[T_{\xi_{0}}\right]$ $\subseteq A$ is superperfect, while $C=A-P$ is $\sigma$-bounded. To show uniqueness notice that if $P, C$ satisfy the above conditions, then

$$
\begin{aligned}
& P=A^{*}=\{\alpha: \forall G(G \text { open } \& \alpha \in G \Rightarrow G \cap A \text { is not } \sigma \text {-bounded })\} \text { and } \\
& C=A-A^{*} \cdot \square
\end{aligned}
$$

REMARK. This argument can be used to give an alternative proof of the result of Friedman mentioned in Remark (2) of $2 \mathrm{~A}$. Indeed let $A=[T]$ with $T$ recursive. In the notation above, assuming towards a contradiction that $A$ contains no nonempty superperfect subset, we have $T_{\xi_{0}}=\varnothing$, so $\xi_{0}$ is recursive since the derivative $J \rightarrow J^{\prime}$ is arithmetical. Then if $\alpha \in A$ there is $\xi<\xi_{0}$ such that $\alpha \in T_{\xi}-T_{\xi+1}$ and thus for some finite sequence $s, N_{s} \cap\left[T_{\xi}\right]$ is nonempty and compact and thus contains a $\Delta_{1}^{1}$ real since $T_{\xi} \in \Delta_{1}^{1}$, a contradiction.

3. The games $\tilde{G}(A)$ and $\tilde{G}_{p}(B)$. 3A. In order to deal more effectively with the present notions we shall associate with each $A \subseteq \Re$ a game $\tilde{G}(A)$ so that I has 
a winning strategy in $\tilde{G}(A)$ iff $A$ contains a nonempty superperfect subset and II has a winning strategy in $\tilde{G}(A)$ iff $A$ is $\sigma$-bounded. The game $\tilde{G}(A)$ is played as follows:

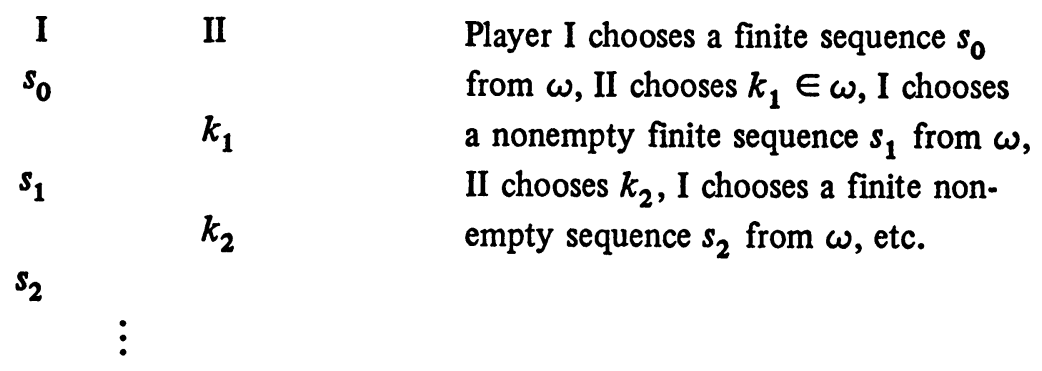

Then I wins iff (i) $\alpha=s_{0}^{-} s_{1}^{-} s_{2} \cdots \in A$ and (ii). For all $m \geqslant 1$, the first member of $s_{m}$ is bigger than $k_{m}$. II wins otherwise.

The following result is the analog of the M. Davis Theorem about the *games, see [D] or [My].

Theorem 3.1. Let $A \subseteq \Re$ and let $\tilde{G}(A)$ be the game described above. Then

(i) I has a winning strategy in $\tilde{G}(A)$ iff $A$ contains a nonempty superperfect set.

(ii) II has a winning strategy in $\tilde{G}(A)$ iff $A$ is $\sigma$-bounded.

Proof. Only the "only if" part of (ii) is nontrivial. So assume II has a winning strategy $\tau$ in $\tilde{G}(A)$. Call a sequence (maybe empty) $\left(s_{0}, k_{1}, s_{1}, k_{2}, \ldots\right.$, $\left.s_{n}, k_{n+1}\right)$ good if for all $1 \leqslant i \leqslant n$ the first member of $s_{i}$ is bigger than $k_{i}$ and for all $0 \leqslant j \leqslant n+1$ the $k_{j}$ 's are determined according to $\tau$. By convention the empty sequence is good. If $\alpha \in A$ there must be some good sequence $u=\left(s_{0}, k_{1}, s_{1}, k_{2}, \ldots, s_{n}, k_{n+1}\right)$ (maybe empty) such that $s_{0}^{-} s_{1}^{-} \cdots s_{n}^{-}$is an initial segment of $\alpha$, say $s_{0}^{-} s_{1}^{-} \cdots s_{n}{ }^{-}=(\alpha(0) \cdots \alpha(m-1))$, and $\alpha(m)>k_{n+1}$ but no good extension of $u$ has this property. Let $K_{u}=\left\{\alpha^{\prime}: s_{0}^{-} s_{1}^{-} \cdots s_{n}^{-}\right.$is an initial segment of $\alpha^{\prime}$, say $\left(\alpha^{\prime}(0) \cdots \alpha^{\prime}(m-1)\right)$, and $\alpha^{\prime}(m)>k_{n+1}$ but for every $s_{n+1} \neq \varnothing$ such that $s_{0}^{\hat{0}} s_{\hat{1}}^{-} \cdots s_{n+1}^{-}$is an initial segment of $\alpha^{\prime}$, say $s_{0}^{\hat{0}} s_{\hat{1}}^{-} \cdots s_{n+1}^{-}$ $=\left(\alpha(0), \ldots, \alpha^{\prime}(l-1)\right)$, we have $\left.\alpha(l) \leqslant k_{n+2}=\tau\left(s_{0}, s_{1}, \ldots, s_{n+1}\right)\right\}$. It is now easy to see that $K_{u}$ is a $\sigma$-compact set. Also $\alpha \in K_{u}$. Since there are only countably many such $K_{u}$ 's, $A$ is $\sigma$-bounded.

COROLLARY 3.2. (i) PD $\Rightarrow$ Every projective set of reals is either $\sigma$-bounded or contains a nonempty superperfect set.

(ii) $\mathrm{AD} \Rightarrow$ Every set of reals is either $\sigma$-bounded or contains a nonempty superperfect set.

3B. In order to get sufficiently strong definability results (having in mind here for example the analog of Martin's Theorem, see [ $\left.\mathbf{M a}_{2}\right]$ that granting PD every countable $\Sigma_{2 n+1}^{1}$ set is contained in some $\left\{\alpha_{m}: m \in \omega\right\}$ where the sequence $\left\{\alpha_{m}\right\}_{m \in \omega}$ is $\Delta_{2 n+1}^{1}$ ) we shall have to consider a modified version of the previous game $\tilde{G}(A)$ which allows for player I to play also witnesses. (The 
same modification of the **-(or Banach-Mazur) games was of course used in the proof of the theorem in $\$ 1$.)

Let $A \subseteq \Re$ and assume that for some set $B \subseteq \Re \times \lambda^{\omega}$, where $\lambda$ is an ordinal,

$$
A=p[B]=\left\{\alpha: \exists f \in \lambda^{\omega}(\alpha, f) \in B\right\} .
$$

Consider the following game $\tilde{G}_{p}(B)$ :

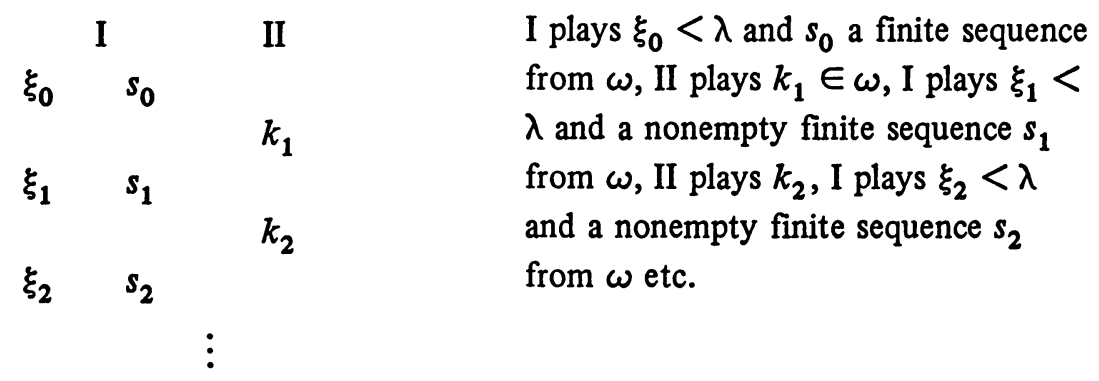

Let $\alpha=s_{0} s_{1} \cdots \in R, f=\left(\xi_{0}, \xi_{1}, \xi_{2}, \ldots\right) \in \lambda^{\omega}$. Then I wins iff $(\alpha, f) \in B$ and for all $i \geqslant 1$, the first member of $s_{i}$ is bigger than $k_{i}$.

It is now easy to prove the following result, using the ideas of the proof of Theorem 3.1 together with the obvious changes suggested by the proof of the theorem in $\S 1$. In its statement we denote by $X^{+}$the smallest admissible set containing $X$. Also $L[X]$ is the universe constructible from $X$.

THEOREM 3.3. Let $\lambda$ be an infinite ordinal and let $B \subseteq R \times \lambda^{\omega}$. Then

(i) If $\delta$ is a winning strategy for $I$ in $\tilde{G}_{p}(B)$ then $A=p[B]$ contains a nonempty superperfect set whose tree belongs in $L[\delta]$.

(ii) If $\mathcal{T}$ is a winning strategy for II in $\tilde{G}_{p}(B)$ then there is a sequence $\left\{\alpha_{\xi}\right\}_{\xi<\lambda} \in \mathcal{I}^{+}$so that for each $\alpha \in A$ there is $\xi<\lambda$, with $\alpha \leqslant \alpha_{\xi}$.

REMARK. In particular, if $\lambda=\omega$ and $\tilde{G}_{p}(B)$ is determined, then $A$ is $\sigma$ bounded $\Leftrightarrow$ II has a winning strategy in $G_{p}(B)$ and $A$ contains a nonempty superperfect set $\Leftrightarrow I$ has a winning strategy in $\tilde{G}_{p}(B)$.

4. Definability results. Using Theorem 3.3, we can now prove without too much effort the next result every part of which is the analog of a well-known fact about countable and perfect sets.

THEOREM. (i) If $A \subseteq \Re$ is $\Sigma_{1}^{1}$, then either $A$ contains a nonempty superperfect set or $A$ is $\sigma$-bounded with a $\Delta_{1}^{1}$ bound.

More generally,

(ii) Determinacy $\left(\Delta_{2 n}^{1}\right) \Rightarrow$ Every $\Sigma_{2 n+1}^{1}$ set either contains a nonempty superperfect set or is $\sigma$-bounded with a $\Delta_{2 n+1}^{1}$ bound.

(iii) If $T$ is a tree on $\omega \times \lambda$, where $\lambda$ is some ordinal, and $A=p[T]$ $=\left\{\alpha: \exists f \in \lambda^{\omega}(\alpha, f) \in[T]\right\}$ then either $A$ contains a nonempty superperfect set 
whose tree is in $L[T]$, or there is a sequence $\left\{\alpha_{\xi}\right\}_{\xi<\lambda}$ in $T^{+}$such that for all $\alpha \in A$ there is $\xi<\lambda$ with $\alpha \leqslant \alpha_{\xi}$.

In particular,

(iv) If $A \subseteq \Re$ is $\Sigma_{2}^{1}$ either $A$ contains a nonempty superperfect set with tree in $L$ or for every $\alpha \in A$ there is $\beta \in L$ with $\alpha \leqslant \beta$, i.e. $A \subseteq\{\alpha: \exists \beta \in L$ ( $\alpha$ $\leqslant \beta)\}$.

Proof. (ii) If $A \in \Sigma_{2 n+1}^{1}$, then $\alpha \in A \Leftrightarrow \exists \beta B(\alpha, \beta)$, where $B \in \Pi_{2 n}^{1}$. The game $\tilde{G}_{p}(B)$ is then a $\Pi_{2 n}^{1}$ game, so it is determined assuming Determinacy $\left(\Delta_{2 n}^{1}\right)$, by Martin [Ma $\mathbf{M}_{1}$. If $A$ contains no nonempty superperfect set then II has a winning strategy in $\tilde{G}_{p}(B)$, thus by Moschovakis [ $\left.\mathbf{M o}_{3}\right]$ II has a $\Delta_{2 n+1}^{1}$ winning strategy. Therefore there is a $\Delta_{2 n+1}^{1}$ bound $\left\{\alpha_{m}\right\}_{m \in \omega}$ for $A$ (i.e. $\alpha(\langle m, l\rangle)=\alpha_{m}(l)$ is $\left.\Delta_{2 n+1}^{1}\right)$.

(iii) The game $\tilde{G}_{p}(B)$, where $B=[T]$, is clearly closed so it is determined. If $I$ has a winning strategy then he has one in $L[T]$, thus $A$ contains a nonempty superperfect set with tree in $L[T]$. If II has a winning strategy then he has one in $T^{+}$and the conclusion follows.

(iv) is clear now since every $\Sigma_{2}^{1}$ set is of the form $p[T]$ for some $T \in L$.

5. Largest $\sigma$-bounded sets in the analytical pointclasses. $5 \mathrm{~A}$. We shall transfer in this section some of the usual results about the structure of countable and thin (i.e. containing no perfect subset) sets in the various analytical pointclasses to the present context. We start with a definition.

Definition. A set $A \subseteq \Re$ is called weakly thin iff it contains no nonempty superperfect set.

Note that if $\tilde{G}(A)$ is determined, $A$ is weakly thin iff $A$ is $\sigma$-bounded. More generally, if $A=p[B]$, and $\tilde{G}_{p}(B)$ is determined, then the same conclusion holds. Thus granting Determinacy $\left(\Delta_{2 n}^{1}\right)$, a set $A \in \Sigma_{2 n+1}^{1}$ is weakly thin iff it is $\sigma$-bounded and granting Determinacy $\left(\Sigma_{2 n+1}^{1}\right)$, a set $A \in \Sigma_{2 n+2}^{1}$ is weakly thin iff it is $\sigma$-bounded. It is consistent with ZFC however that there is a $\Pi_{1}^{1}$ weakly thin set which is a scale, so it is not $\sigma$-bounded. This is the set $\mathfrak{B}_{1}$ defined below.

5B. Our immediate goal is to show that there are largest weakly thin $\Pi_{2 n+1}^{1}$ and $\Sigma_{2 n+2}^{1}$ sets granting appropriate determinacy hypotheses for $n \geqslant 1$. The general abstract approach of $\S 1$ of $\left[\mathrm{Ke}_{2}\right]$ is particularly useful here. By Theorem (1A-2) of $\left[\mathbf{K e}_{2}\right]$ it is enough to prove that the class of weakly thin sets has an appropriate additivity and satisfies some definability conditions. This is accomplished in the following two lemmas.

LEMmA 5.1. Assume $n \geqslant 0$ and Determinacy $\left(\Delta_{2 n}^{1}\right)$. Then, the class of weakly thin sets is $\Sigma_{2 n+1}^{1}$ and $\Pi_{2 n+1}^{1}$ additive. Similarly, if Determinacy $\left(\Sigma_{2 n+1}^{1}\right)$ holds the class of weakly thin sets in $\Sigma_{2 n+2}^{1}$ and $\Pi_{2 n+2}^{1}$ additive.

Proof. For the definitions see p. 265 of $\left[\mathrm{Ke}_{2}\right]$. Consider the first assertion; the other can be proved by exactly the same argument. Let $\Gamma=$ Boolean 
algebra generated by $\Sigma_{2 n+1}^{1}$. We shall prove (the stronger result) that the class of weakly thin sets is $\Gamma$-additive. So assume $\left\{A_{\xi}\right\}_{\xi<\theta}$ is a sequence of sets of reals such that each $A_{\xi}$ is weakly thin and the prewellordering on $\bigcup_{\xi<\theta} A_{\xi}$ given by

$$
x \leqslant y \Leftrightarrow \mu \xi\left(x \in A_{\xi}\right) \leqslant \mu \xi\left(y \in A_{\xi}\right)
$$

is in $\Gamma$. We have to prove that $\bigcup_{\xi<\theta} A_{\xi}$ is also weakly thin. The proof is by induction on $\theta \geqslant 2$ the case $\theta=2$ being obvious. Consider the successor case first: Let $\theta=\eta+1$. The prewellordering

$$
x \leqslant y \Leftrightarrow x, y \in \bigcup_{\xi<\eta} A_{\xi} \& \mu \xi\left(x \in A_{\xi}\right) \leqslant \mu \xi\left(y \in A_{\xi}\right)
$$

is clearly in $\Gamma$ since

$$
x \leqslant y \Leftrightarrow x \leqslant y \& \neg\left(x_{0} \leqslant x\right) \& \neg\left(x_{0} \leqslant y\right),
$$

where $x_{0}$ is a fixed element of $A_{\eta}-\cup_{\xi<\eta} A_{\xi}$ (if no such $x_{0}$ exists the result is obviously true). By induction hypothesis $A=\cup_{\xi<\eta} A_{\xi}$ is weakly thin. Let $B=A_{\eta}$. Then $B$ is also weakly thin. Assume towards a contradiction that $A \cup B$ is not weakly thin. Then $A \cup B$ contains a superperfect set $P$ homeomorphic to $\Re$. Then $A \cap P$ must be meager in $P$ (with the relative topology). Otherwise $A \cap P$, having as it does the property of Baire, is comeager in an open set $G$ of $P$. Thus $A \cap P$ contains a $G_{\delta}$ set $D$ dense in $G$. But $D$ cannot be $\sigma$-bounded so $D$ contains a superperfect set and thus so does $A$, a contradiction. Similarly $B \cap P$ is meager in $P$. Thus $P$ is meager in $P$, contradicting the Baire Category Theorem.

For the limit case, assume towards a contradiction, that $P \subseteq \cup_{\xi<\theta} A_{\xi}$ is a superperfect set homeomorphic to $\Re$. Consider the relation

$$
x \leqslant " y \Leftrightarrow x \leqslant y \& x, y \in P .
$$

Then $\leqslant "$ is a prewellordering on $P$ which is in $\Gamma$, so has the property of Baire. For each $y \in P,\left\{x: x \leqslant{ }^{\prime \prime} y\right\}=\bigcup\left\{A_{\eta}: \eta \leqslant \mu \xi\left(y \in A_{\xi}\right)\right\} \cap P$, so by the previous arguments and the induction hypothesis $\{y: x \leqslant$ | $y\}$ is meager in $P$. So by the Kuratowski-Ulam Theorem (see [O, p. 56]), which is the analog of Fubini for category, we must also have that on a comeager in $P$ set of $x$ 's $\{y: x \leqslant 1$ " $y$ is also meager in $P$. Thus $P$ is meager in $P$, a contradiction.

LEMMA 5.2. Assume $n \geqslant 0$ and Determinacy $\left(\Delta_{2 n}^{1}\right)$. Let $G \subseteq \Re \times \Re$ be $\Pi_{2 n+1}^{1}$ and R-universal for $\Pi_{2 n+1}^{1}$ subsets of $\Re_{\text {. Then if }} G_{\alpha}=\{\beta:(\alpha, \beta) \in G\}$, the set $W^{2 n+1}(\alpha) \Leftrightarrow \Re-G_{\alpha}$ is weakly thin is $\Pi_{2 n+1}^{1}$.

Proof. Let $A \subseteq \Re$ be $\Sigma_{2 n+1}^{1}$. Let $B \in \Pi_{2 n}^{1}$ be such that $\alpha \in A \Leftrightarrow \exists \beta$ $B(\alpha, \beta)$. Then, $A$ is weakly thin $\Leftrightarrow$ II has a winning strategy in $\tilde{G}_{p}(B) \Leftrightarrow \mathrm{I}$ has no winning strategy in $\tilde{G}_{p}(B)$. Since $\tilde{G}_{p}(B)$ is a $\Pi_{2 n}^{1}$ game this statement is 
$\Pi_{2 n+1}^{1}$, uniformly in a code for $B$ and we are done.

5C. Using this lemma and Theorem (1A-2) from $\left[\mathrm{Ke}_{2}\right]$ we immediately have

TheOREM 5.3. (i) There is a largest weakly thin $\Pi_{1}^{1}$ set.

(ii) Assume $n>0$ and Determinacy $\left(\Delta_{2 n}^{1}\right)$. There exists a largest weakly thin $\Pi_{2 n+1}^{1}$ set.

In particular, granting PD, there is for each $n \geqslant 0$ a largest $\sigma$-bounded $\Pi_{2 n+1}^{1}$ set.

We also have the corresponding result for even levels.

THEOREM 5.4. (i) Assume there are only countably many constructible reals. Then there is a largest $\sigma$-bounded $\Sigma_{2}^{1}$ set, namely $\{\alpha: \exists \beta \in L(\alpha \leqslant \beta)\}$.

(ii) Assume $n \geqslant 1$ and Determinacy $\left(\Sigma_{2 n+1}^{1}\right)$. Then there is a largest $\sigma$-bounded $\Sigma_{2 n+2}^{1}$ set.

PRoof. (i) follows from the theorem in $\$ 4$.

(ii) Let $P(n, \alpha, \beta)$ be $\Pi_{2 n+1}^{1}$ and $\omega$-universal for $\Pi_{2 n+1}^{1}$ subsets of $\Re \times \Re$. By the uniformization theorem (see $\left.\left[\mathrm{Mo}_{4}\right]\right)$ find $P^{*}(n, \alpha, \beta)$ uniformizing $P(n, \alpha, \beta)$ on $\beta$. Let $\varphi$ be a $\Pi_{2 n+1}^{1}$-norm on $P$ (for the definition see for example $\left[\mathbf{M o}_{4}, p\right.$. 733]). Consider the set $B$ given by

$\alpha \in B \Leftrightarrow \exists n \exists \beta\left[P^{*}(n, \alpha, \beta)\right.$

$\&\left\{\alpha^{\prime}: \exists \beta^{\prime}\left(P^{*}\left(n, \alpha^{\prime}, \beta^{\prime}\right) \& \varphi\left(n, \alpha^{\prime}, \beta^{\prime}\right) \leqslant \varphi(n, \alpha, \beta)\right)\right\}$ is $\sigma$-bounded].

By Lemma 5.2, $B$ is $\Sigma_{2 n+2}^{1}$ and it is trivial to check that if $A \subseteq \Re$ is $\Sigma_{2 n+2}^{1}$ and $\sigma$-bounded then $A \subseteq B$. So it is enough to prove that $B$ itself is $\sigma$-bounded. Let $Q_{n}(\alpha, \beta) \Leftrightarrow P^{*}(n, \alpha, \beta) \&\left\{\alpha^{\prime}: \exists \beta^{\prime}\left(P^{*}\left(n, \alpha^{\prime}, \beta^{\prime}\right) \& \varphi\left(n, \alpha^{\prime}, \beta^{\prime}\right) \leqslant \varphi(n, \alpha, \beta)\right)\right\}$ is $\sigma$-bounded, and $B_{n}=\left\{\alpha: \exists \beta Q_{n}(\alpha, \beta)\right\}$. Since $B=\cup_{n} B_{n}$ it is enough to show each $B_{n}$ is $\sigma$-bounded. Let $\theta=\sup \left\{\varphi(n, \alpha, \beta): P^{*}(n, \alpha, \beta)\right\}$ and for $\xi<\theta$ let $A_{\xi}=\left\{\alpha: \exists \beta\left(Q_{n}(\alpha, \beta) \& \varphi(\alpha, \beta)=\xi\right)\right\}$. Then $B_{n}=\cup_{\xi<\theta} A_{\xi}$ and the prewellordering $\alpha_{1} \leqslant \alpha_{2} \Leftrightarrow \alpha_{1}, \alpha_{2} \in B_{n} \& \mu \xi\left(\alpha_{1} \in A_{\xi}\right) \leqslant \mu \xi\left(\alpha_{2} \in A_{\xi}\right)$ $\Leftrightarrow \exists \beta_{1} \exists \beta_{2}\left(Q_{n}\left(\alpha_{1}, \beta_{1}\right) \& Q_{n}\left(\alpha_{2}, \beta_{2}\right) \& \varphi\left(n, \alpha_{1}, \beta_{1}\right) \leqslant \varphi\left(n, \alpha_{2}, \beta_{2}\right)\right)$ is $\Sigma_{2 n+2}^{1}$. Since each $A_{\xi}$ is $\sigma$-bounded, we conclude by Lemma 5.1 that $B_{n}=\cup_{\xi<\theta} A_{\xi}$ is also $\sigma$-bounded and we are done.

NotATiON. Let $\mathscr{B}_{1}$ be the largest weakly thin $\Pi_{1}^{1}$ set, $\mathscr{B}_{2}=\{\alpha: \exists \beta$ $\in L(\alpha \leqslant \beta)\}$ and granting PD let $\mathscr{B}_{n}=$ largest $\sigma$-bounded $\Sigma_{n}^{1}$ or $\Pi_{n}^{1}$ set according as $n \geqslant 3$ is even or odd.

REMARKS. (1) It is easy to see, using PD, that there are no largest $\sigma$-bounded $\Sigma_{2 n+1}^{1}$ or $\Pi_{2 n}^{1}$ sets, $n \geqslant 0$. This is because a $\sigma$-bounded set is meager and every comeager $\Pi_{2 n+1}^{1}$ or $\Sigma_{2 n}^{1}$ set contains (by $\left[\mathrm{Ke}_{3}\right]$ and $\left[\mathrm{Mo}_{4}\right]$ ) a $\Delta_{2 n+1}^{1}$ or $\Delta_{2 n}^{1}$ real, respectively.

(2) Let for $n \geqslant 1$, using PD, $e_{n}$ be the largest countable $\Pi_{n}^{1}$ or $\Sigma_{n}^{1}$ set, according as $n$ is odd or even (see [ $\left.\left.\mathbf{K e}_{2}\right]\right)$ and $\mathfrak{\pi}_{n}=$ largest meager $\Pi_{n}^{1}$ or $\Sigma_{n}^{1}$ set, according as $n$ is again odd or even (see [Ke $]$ or [St]). Then clearly 


$$
\mathfrak{C}_{n} \subsetneq \mathfrak{B}_{n} \subsetneq \mathfrak{N}_{n} .
$$

5D. We shall concentrate now on the set $\mathscr{B}_{1}=$ largest weakly thin $\Pi_{1}^{1}$ set. Clearly $\mathfrak{B}_{1} \subseteq \mathscr{T}_{2}$ so for each $\alpha \in \mathscr{B}_{1}$ there is $\beta \in L$ such that $\alpha \leqslant \beta$. In analogy with the Guaspari-Sacks characterization of $C_{1}$ as $\left\{\alpha: \alpha \in L_{\omega_{1}^{\alpha}}\right\}$ (see [Gu] or [ $\left[\mathbf{K e}_{2}\right]$, Theorem (2A-1)), where of course $\omega_{1}^{\alpha}=$ least ordinal nonrecursive in $\alpha$, we have:

THEOREM 5.5. The largest weakly thin $\Pi_{1}^{1}$ set is equal to $\left\{\alpha: \exists \beta\left(\beta \in L_{\omega \mathrm{f}}^{\alpha} \& \alpha\right.\right.$ $\leqslant \beta)\}$.

Proof. Use the same idea as in the proof of Theorem (2A-1) in $\left[\mathrm{Ke}_{\mathbf{2}}\right]$, together with the Theorem in $\$ 4$ (part iii) and the category arguments in Lemma 5.1 to replace the measure theoretic ones used in Theorem (2A-1) of $\left[\mathrm{Ke}_{2}\right]$.

Similarly we have the following analog of Theorem (2A-2) in [ $\left.\mathbf{K e}_{2}\right]$, letting $\alpha \leqslant_{n}^{b} \beta \Leftrightarrow \exists \gamma \in \Delta_{n}^{1}(\beta)(\alpha \leqslant \gamma)$.

THEOREM 5.6. For all $\alpha$,

$$
\alpha \in \mathscr{B}_{1} \Leftrightarrow \forall \beta\left(\omega_{1}^{\alpha} \leqslant \omega_{1}^{\beta} \Rightarrow \alpha \leqslant_{1}^{b} \beta\right) .
$$

PROoF. The direction $\Rightarrow$ is similar to the corresponding part in the proof of Theorem (2A-2) in $\left[\mathrm{Ke}_{2}\right]$. For the proof of $\Leftarrow$ it is enough to show that $B=\left\{\alpha: \forall \beta\left(\omega_{1}^{\alpha} \leqslant \omega_{1}^{\beta} \Rightarrow \alpha \leqslant 1 \beta\right)\right\}$ is $\Pi_{1}^{1}$ and weakly thin. It is clearly $\Pi_{1}^{1}$. To show it is weakly thin assume $P \subseteq B$ is a superperfect set homeomorphic to R. Consider the prewellordering

$$
\alpha \leqslant \beta \Leftrightarrow \alpha, \quad \beta \in P \& \omega_{1}^{\alpha} \leqslant \omega_{1}^{\beta}
$$

on $P$. For each $\beta \in P,\{\alpha: \alpha \leqslant \beta\}=\left\{\alpha \in P: \omega_{1}^{\alpha} \leqslant \omega_{1}^{\beta}\right\}$ is contained in a $\sigma$ compact set, namely $\{\alpha: \alpha \leqslant 1\}$. So $\{\alpha: \alpha \leqslant \beta\}$ is meager in $P$ and this contradicts as usual the Kuratowski-Ulam Theorem.

$5 \mathrm{E}$. We shall conclude this section with some remarks on $\mathscr{B}_{2}=\{\alpha: \exists \beta$ $\in L(\alpha \leqslant \beta)\}$ and some open problems. We have seen in Theorem 5.4 that, granting $\Re \cap L$ is countable, $\mathscr{B}_{2}$ is the largest $\sigma$-bounded $\Sigma_{2}^{1}$ set. Of course we could only assume that $R \cap L$ was $\sigma$-bounded. In this case the converse also holds: If there is a largest $\sigma$-bounded $\Sigma_{2}^{1}$ set then $\Re \cap L$ is $\sigma$-bounded. This is because every $\Sigma_{2}^{1}$ set which contains all the countable $\Sigma_{2}^{1}$ sets must contain also $\Re \cap L$. (Otherwise let $\alpha_{0}$ be the least element of $L$ not in the given $\Sigma_{2}^{1}$ set $A$ which satisfies the above. Then by a result of Friedman $\left[\mathrm{Fr}_{2}\right], \alpha_{0}$ is a $\Pi_{2}^{1}$ singleton in $L$, so the set of reals in $L$ preceding or being equal to $\alpha_{0}$ is a countable $\Sigma_{2}^{1}$ set not contained in $A$, a contradiction.) It seems relevant to mention also that according to unpublished results of Solovay (see [He]), in the model $M$ of set theory obtained from $L$ by adding (say) $\aleph_{2}$ random reals, 
$\Re \cap L$ is weakly thin and thus so is $\mathscr{B}_{1}$, but $\mathcal{C}_{1}$ and thus $\mathscr{B}_{1}$ is a scale

$$
\text { (so }\left\{\alpha: \exists \beta \in \mathcal{C}_{1}(\alpha \leqslant \beta)\right\}=\mathscr{B}_{2}=\mathscr{R} \text { ). }
$$

In particular, $\mathscr{B}_{1}$ is not $\sigma$-bounded which of course is also true in $L$. (This supplies a proof for the remark immediately preceding $5 \mathrm{~B}$.)

By Theorem 5.4 there is a largest $\sigma$-bounded $\Sigma_{2 n}^{1}$ set $\mathscr{B}_{2 n}$ for each $n \geqslant 1$, granting PD. Since $L \cap \Re=e_{2}=$ largest countable $\Sigma_{2}^{l}$ set, the fact that $\mathscr{B}_{2}=\left\{\alpha: \exists \beta \in \mathcal{C}_{2}(\alpha \leqslant \beta)\right\}$ suggests the following

Conjecture. Assuming PD, we have for each $n \geqslant 1$

$$
\mathscr{B}_{2 n}=\left\{\alpha: \exists \beta \in \mathcal{C}_{2 n}(\alpha \leqslant \beta)\right\} \text {. }
$$

We also do not know if there is any relationship of the above type between $\mathcal{C}_{2 n+1}$ and $\mathscr{B}_{2 n+1}$ for $n \geqslant 0$. Since $\alpha \in \mathscr{B}_{2} \Leftrightarrow \exists \beta \in \Delta_{2}^{1}(\alpha)\left(\beta \in \mathcal{C}_{2} \& \alpha \leqslant \beta\right)$ one might wonder about the validity of the following formula (granting PD for $n \geqslant 1)$

$$
\alpha \in \mathscr{B}_{2 n+1} \Leftrightarrow \exists \beta \in \Delta_{2 n+1}^{1}(\alpha)\left(\beta \in \mathcal{C}_{2 n+1} \& \alpha \leqslant \beta\right) .
$$

This is however an open problem.

6. Generalizations; the games $G^{\mathcal{E}}(A)$. In this last section we shall present a generalization of the ${ }^{*}$ - and ${ }^{* *}$-games (see [My]) as well as the $\sim$-games considered in the present paper. The generalized games we have in mind give a corresponding notion of smallness for sets of reals whose special cases are countability, being of the first category and $\sigma$-boundedness. We shall also see how the main results of the previous sections generalize to this wider context.

Let $X$ be an arbitrary set having more than one element $(X=\{0,1\}$ or $X=\omega$ are essentially the only cases we are interested in here). Let also $R$ be an arbitrary nonempty set, whose elements we shall call requirements and let $S$ be a function which assigns to each $r \in R$ a nonempty set of nonempty finite sequences from $X$, i.e. $S: R \rightarrow$ power $\left(X_{*}^{<\omega}\right)-\{\varnothing\}$, where $X_{*}^{<\omega}$ $={ }^{\text {def }} \cup_{n \geqslant 1} X^{n}$. If $u \in S(r)$ we shall say that $u$ satisfies the requirement $r$ and we shall write for convenience $u \prec_{s} r$ in this case. We shall assume that $u \prec_{s} r$ satisfies the following conditions (of which the second is essentially a nontriviality condition that will assure us later that in the notion of smallness generated by $\langle R, S\rangle$ singletons are small):

(1) $v \prec u \& u \prec_{S} r \Rightarrow v \prec_{S} r$ (i.e. any extension of a sequence that satisfies $r$ satisfies $r$ also),

(2) $\forall x \in X \exists r \in R \forall u \in X_{*}^{<\omega}\left(u \prec_{S} r \Rightarrow u(0) \neq x\right)$ (i.e. for every $x \in X$ there is a requirement which forces the first member of any sequence satisfying it to be different than $x$ ).

(3) There is $l: R \rightarrow \omega$ such that if $r \in R, u \in X_{*}^{<\omega}$ and $\exists u^{\prime} \leqslant u\left(u^{\prime} \prec_{S} r\right)$ but $u K_{S} r$, then there is $q \in R$ with $l(q)<l(r)$ such that $u v \prec_{S} r$ iff $v \prec_{S} q$. 
ExAmples. (E1) $X=\{0,1\}, R=\{0,1\}, u \prec_{S} r \Leftrightarrow u(0)=r$ (here $u(0)=$ first member of $u) . l(r) \equiv 0$.

(E2) $X$ arbitrary, $R=X^{<\omega}, u \prec_{S} r \Leftrightarrow u<r$. $l(r)=$ length $(r)$.

(E3) $X=\omega, R=\omega, u \prec_{S} r \Leftrightarrow u(0)>r . l(r) \equiv 0$.

Suppose now a pair $\mathcal{E}=\langle R, S\rangle$ satisfying the above conditions is given. Abusing language we shall call $\mathcal{E}$ a requirement set. To each $A \subseteq X^{\omega}$ we associate the following game $G^{\mathscr{E}}(A)$ :

\begin{tabular}{|c|c|}
\hline II & I plays a finite sequence $u_{0}$ from $X$, \\
\hline$r_{1}$ & $\begin{array}{l}\text { II plays } r_{1} \in R \text {, I plays a nonempty } \\
\text { finite sequence } u_{1} \text { from } X \text {, II plays }\end{array}$ \\
\hline$r_{2}$ & $\begin{array}{l}r_{2} \in R, \text { I plays a nonempty finite } \\
\text { sequence } u_{2} \text { from } X \text {, etc. }\end{array}$ \\
\hline
\end{tabular}

Let $f=\hat{u_{0}} \hat{u_{1}} \overrightarrow{u_{2}} \cdots \in X^{\omega}$. Then I wins iff (i) $f \in A$ and (ii) $\forall i \geqslant 1, u_{i} \prec_{s} r_{i}$. Otherwise II wins.

ExAMPLES. In Example E1 before, clearly $G^{\mathscr{E}}(A)$ is equivalent to the game $G^{*}(A)$. In E2, $G^{\mathcal{E}}(A)$ is equivalent to $G^{* *}(A)$. In E3, $G^{\mathcal{E}}(A)$ is equivalent to $\tilde{G}(A)$.

Definition. Let $A \subseteq X^{\omega}$ be a closed set. We call $A$ E-nowhere dense if for each $u \in T_{A}$ there is a requirement $r \in R$ such that for all $v \in X_{*}^{<\omega}, u v$ $\in T_{A} \Rightarrow u K_{S} r$. We call a set $A \subseteq X^{\omega}$ E-meager if it is contained in a countable union of closed $\mathcal{E}$-nowhere dense sets.

Examples. In E1, $A \subseteq 2^{\omega}$ is closed $\delta$-nowhere dense iff $A$ is a singleton. Thus $A \subseteq 2^{\omega}$ is -meager iff $A$ is countable. In E2, $A \subseteq X^{\omega}$ is closed \&nowhere dense iff $A$ is closed nowhere dense. Thus $A \subseteq X^{\omega}$ is $\mathcal{E}$-meager iff $A$ is meager. Finally in E3, $A \subseteq \omega^{\omega}$ is closed $\mathcal{E}$-nowhere dense iff $A$ is compact and so $A \subseteq \omega^{\omega}$ is $\varepsilon$-meager iff $A$ is $\sigma$-bounded.

Clearly "\&-meager" is the notion of smallness associated with $\mathcal{E}$. Notice that because of condition (ii) in the definition of a requirement set every singleton in $\delta$-nowhere dense. So every countable set is \&-meager. Also every $\delta$-nowhere dense closed set is nowhere dense and so any $\&$-meager set is meager. We shall now define a notion of "extreme largeness" opposite to the notion of "Emeager".

Definition. Let $J$ be a tree on $X^{<\omega}=\cup_{n} X^{n}=$ the set of all finite sequences from $X$. We shall call $J$ E-perfect if the following two conditions are met:

(i) For each $\phi \neq p \in J,\left\{u \in X_{*}^{<\omega}: p^{-}(u) \in J\right\}$ is E-dense i.e. for each $r \in R$ there is a $u \in X_{*}^{<\omega}$ and $u^{\prime} \preccurlyeq u$ such that $p^{\prime}(u) \in J$ and $u^{\prime} \prec_{S} r$.

(ii) For each $p \in J$ if $p^{-}(u) \in J, p^{-}(v) \in J$ and $u \neq v$ then $u, v$ are incompatible. 
Now for each $\delta$-perfect tree $J$ we let $[J]=\left\{f \in X^{\omega}\right.$ : for all $n$ there is $s_{0}, s_{1}, \ldots, s_{n}$ such that $\left(s_{0}, s_{1}, \ldots, s_{n}\right) \in J$ and $s_{0}{ }^{-} s_{1} \cdots s_{n}{ }^{-}$is an initial segment of $f\}\left({ }^{2}\right)$. (Note here that $s_{0}, s_{1}, \ldots, s_{n}$ must be necessarily unique.) We call a set $A \subseteq X^{\omega}$ E-perfect if $A=[J]$ for some $\delta$-perfect tree $J$.

It is now easy to see that every $\delta$-perfect set is a $G_{\delta}$ in the space $X^{\omega}$. On the other hand if $\mathcal{E}$ has the property that

$$
u \prec_{s} r \Leftrightarrow(u(0)) \prec_{s} r
$$

(i.e. satisfaction of a requirement depends only on the first member of a sequence), which is obviously the case in Examples E1 and E3 above, then every $\delta$-perfect set contains a closed $\delta$-perfect subset.

EXAMPLES. In El every nonempty $\delta$-perfect set contains a nonempty perfect subset. In E2 a nonempty E-perfect set is a dense in some nonempty neighborhood $G_{\delta}$ set. In E3 a nonempty $\mathcal{E}$-perfect set contains a nonempty superperfect subset.

We now have the analog of Theorem 3.1, provided $X$ is countable and also $R$ is countable, in which case (abusing language again) we shall say that " $\mathscr{E}$ is countable". It reads as follows: Let $\delta$ be a countable requirement set on a countable set $X$ and $A \subseteq X^{\omega}$. Then I has a winning strategy in $G^{\mathscr{E}}(A)$ iff $A$ contains a nonempty $\mathcal{E}$-perfect set and II has a winning strategy in $G^{\mathscr{E}}(A)$ iff $A$ is $\delta$-meager. (Again the only nontrivial thing to prove is that if II has a winning strategy $\mathcal{J}$ then $A$ is $\mathcal{E}$-meager. For that call a sequence $p=\left(u_{0}, r_{1}\right.$, $\left.u_{1}, \ldots, u_{n}, r_{n+1}\right)$, where $u_{0} \in X^{<\omega}, u_{i} \in X_{*}^{<\omega}$ for $i>0$ and $r_{i} \in R$, good if for all $i \geqslant 1, u_{i} \prec_{s} r_{i}$ and the $r_{i}$ are determined following $\mathcal{T}$. By convention the empty sequence is good. Given $f \in A$ call $p$ good for $f$ if there is $u_{n+1}$ such that $u_{0} u_{1}^{-} \cdots u_{n}^{-} u_{n+1}$ is an initial segment of $f$ and $u_{n+1} \prec_{s} r_{n+1}$. Thus the empty sequence is automatically good for $A$. Now there must be some $p$ (as above) good for $f$ which has no proper extension good for $f$. Then $f \in K_{p}=\left\{f^{\prime}: p\right.$ is good for $f^{\prime}$ but for no $\left(u_{n+1}, r_{n+2}\right)$ such that $p^{-}\left(u_{n+1}, r_{n+2}\right)=q$ is good, $q$ is also good for $\left.f^{\prime}\right\}$. Clearly $K_{p}$ is a $\delta$-meager set. Since there are only countable many $K_{p}$ 's and $A$ is contained in their union, $A$ must be $\delta$-meager.) We can also get immediately the generalization of Corollary 3.2.

The next step is to consider the analog of Theorem 3.3. This is completely straightforward, using the ideas in the proof of the theorem in $\$ 1$, after one defines the game $G_{p}^{\mathcal{E}}(B)$ for $B \subseteq X^{\omega} \times \lambda^{\omega}$ in the obvious fashion. From that the definability results of $\S 4$ follow immediately. For simplicity take $X \subseteq \omega$ to be a recursive set now and assume that both $R, \prec_{S}$ are recursive (coding them appropriately if necessary as in the case of E2 for instance). Then, for example,

(2) This use of $[\mathbf{J}]$ is slightly different from the one in 1C. Which one we use will be clear from the context and the convention that for the rest of this paper $J_{s}$ will be used for trees on $X^{<\omega}$ while $T$ s will be used for trees on $X$. 
the analog of part (i) of the theorem in $\S 4$ reads: If $A \subseteq X^{\omega}$ is $\Sigma_{1}^{1}$ then either $A$ contains a nonempty $\mathcal{E}$-perfect set or $A \subseteq \cup_{n}\left[T_{n}\right]$ where each $\left[T_{n}\right]=F_{n}$ is closed \&-nowhere dense and $P(s, n) \Leftrightarrow s \in T_{n}$ is $\Delta_{1}^{1}$. Also (iv) reads: If $A$ is $\Sigma_{2}^{1}$ then either $A$ contains a nonempty $\varepsilon$-perfect set with tree in $L$ or every $\alpha \in A$ is contained in some closed E-nowhere dense set with tree in $L$.

We now proceed to consider the generalizations to the present context of the results in $\S 5$.

Definition. Let $X \subseteq \omega$ and $\mathcal{E}$ be a countable requirement set on $X$. A set $A \subseteq X^{\omega}$ is called $\mathcal{E}$-thin if it contains no nonempty $\mathcal{E}$-perfect subset.

Again if $G^{\mathcal{E}}(A)$ is determined, $A$ is $\mathcal{E}$-thin iff $A$ is $\mathcal{E}$-meager. Lemmas 5.1 and 5.2 go again through easily. (For example consider Lemma 5.1. Following the pattern of its proof as well as its notation assume $\left\{A_{\xi}\right\}_{\xi<\theta}$ is a sequence of $\mathcal{E}$ thin sets such that $f \leqslant g \Leftrightarrow \mu \xi\left(f \in A_{\xi}\right) \leqslant \mu \xi\left(g \in A_{\xi}\right)$ is in $\Gamma$ (here $f, g$ vary over $\left.X^{\omega}\right)$ and consider for simplicity the first case where $\theta=\eta+1$. We can conclude exactly as before that $A=\cup_{\xi \leqslant \eta} A_{\xi}$ is \&-thin by induction hypothesis. Put $B=A_{\eta}$. If $A \cup B$ was not $\mathcal{E}$-thin let $P=[J] \subseteq A \cup B$ be an $\mathcal{E}$ perfect set. It will be enough to show that $A \cap P, B \cap P$ are meager in $P$ (then $P$ would be meager in $P$, which contradicts the fact that $P$ being a $G_{\delta}$ of $X^{\omega}$ is topologically complete (see [O, p. 47]) so satisfies Baire's Theorem). Take $A$ for example. Since $A \cap P$ has the property of Baire in $P$, if it is not meager in $P$ it musi be comeager in some neighborhood $N_{u} \cap P$ (where $u \in X^{<\omega}$ ) of $P$. Then $A \cap P$ contains a dense in $N_{u} \cap P G_{\delta}$ subset $D$ of $P$. But then $I$ has a winning strategy in the game $G^{\mathcal{E}}(D)$, so $\mathrm{I}$ has a winning strategy in $G^{\mathscr{E}}(A)$ which contradicts the assumption that $A$ is $\mathcal{E}$-thin.) Using the generalizations of Lemmas 5.1 and 5.2 and assuming again that $X, R, \prec_{S}$ are recursive we can show immediately the existence of a largest $\varepsilon$-thin $\Pi_{1}^{1}$ set and assuming PD, we can also show the existence of largest $\delta$-thin (or equivalently $\delta$-meager) $\Pi_{n}^{1}$ or $\Sigma_{n}^{1}$ sets, according as $n$ is odd or even. We shall denote these sets by $\pi_{n}^{\varepsilon^{n}}$. Clearly $e_{n} \subseteq \Re_{n}^{\mathcal{E}} \subseteq \Re_{n}$ for every $\mathcal{E}$. Finally letting for each $M$ and each $f \in X^{\omega}$,

$$
f \mathcal{E} M \Leftrightarrow \exists T \in M([T] \text { is } \mathcal{E} \text {-nowhere dense \& } f \in[T])
$$

it is straightforward to check that

$$
\pi_{2}^{\mathcal{E}}=\left\{f \in X^{\omega}: f \mathcal{E} L\right\} \quad \text { and } \mathscr{N}_{1}^{\mathcal{E}}=\left\{f \in X^{\omega}: f \mathcal{E} L_{\omega}\right\} .
$$

Finally letting $f \leqslant_{n}^{\mathcal{E}} g \Leftrightarrow \exists T \in \Delta_{n}^{1}(g)(f \in[T] \&[T]$ is \&-nowhere dense) we have $f \in \Re_{1}^{\mathcal{E}} \Leftrightarrow \forall g\left(\omega_{1}^{f} \leqslant \omega_{1}^{g} \Rightarrow f \leqslant \leqslant_{1}^{\mathcal{E}} g\right)$.

ADDED IN PROOF. St. Raymond has independently proved the part of Corollary 3.2(i) which states that a $\Sigma_{1}^{1}$ set of reals is either $\sigma$-bounded or contains a nonempty superperfect set; see J. St. Raymond, Un théorème d'approximation par l'intérieur, C. R. Acad. Sci. Paris (Juin 1975). Louveau ( $\sigma$-ideaux engendres par des ensembles fermes et théorèmes d'approximation (to 
appear) has obtained generalizations of the results in $\$ \S 2-5$ of our paper along directions different than those of $\S 6$. We would like to thank A. Louveau for pointing out a number of errors in a preprint of the present paper.

\section{REFERENCES}

[Bu] D. R. Busch, Some problems connected with the axiom of determinacy, $\mathrm{Ph} . \mathrm{D}$. Thesis, Rockefeller Univ., 1972.

[D] M. Davis, Infinite games of perfect information, Advances in Game Theory, Ann. of Math. Studies, no. 52, Princton Univ. Press, Princeton, N.J., 1964, pp. 85-101. MR 30 \# 965.

[Fe] J. E. Fenstad, The axiom of determinateness, Proc. Second Scandinavian Logic Sympos., North-Holland, Amsterdam, pp. 41-61.

[Fr $\mathbf{F}_{1}$ H. Friedman, Borel sets and hyperdegrees, J. Symbolic Logic 38 (1973), 405-409. MR 49 $\# 30$.

$\left[\mathrm{Fr}_{2}\right] \longrightarrow, A$ basis theorem for $L$ (circulated notes).

[Gu] D. Guaspari, Thin and wellordered analytical sets, Ph.D. Thesis, Univ. of Cambridge, 1972.

[H] S. Hechler, On the existence of certcin cofinal subsets of ${ }^{\omega} \omega$, Proc. Sympos. Pure Math., vol. 13, part 2, Amer. Math. Soc., Providence, R.I., 1974, pp. 155-173. MR 50 \# 12716.

[Ke $\left.{ }_{1}\right]$ A. S. Kechris, Lecture notes on descriptive set theory, M.I.T., Cambridge, Mass., 1973.

$\left[\mathrm{Ke}_{2}\right]$ - The theory of countable analytical sets, Trans. Amer. Math. Soc. 202 (1975), 259-298.

[Ke $\left.{ }_{3}\right]-$ Measure and category in effective descriptive set theory, Ann. Math. Logic 5 (1972/73), 337-384. MR 51 \#5308.

[Ku] K. Kuratowski, Topology, Vol. 1, Academic Press, New York, 1966. MR 36 \#840.

$\left[\mathrm{Ma}_{1}\right]$ D. A. Martin, $\Delta_{2 n}^{1}$ determinacy implies $\Sigma_{2 n}^{1}$ determinacy, 1973 (circulated notes).

[Ma $\mathrm{Ma}_{2} \longrightarrow$, Countable $\Sigma_{2 n+1}^{1}$ sets, 1972 (circulated notes).

[Mo $]$ Y. N. Moschovakis, Descriptive set theory, North-Holland (to appear).

[Mo $\left.\mathrm{Mo}_{2}\right]$ - Determinacy and prewellorderings of the continuum, Mathematical Logic and Foundations of Set Theory (Proc. Internat. Colloq., Jerusalem, 1968), North-Holland, Amsterdam, 1970, pp. 24-62. MR 43 \#6082.

[Mo $]$, Analytical definability in a playful universe, Proc. Fourth Internat. Congr. Logic, Methodology and Philosophy of Science, (Bucharest, 1971), North-Holland, Amsterdam, 1973.

[Mo $]$, Uniformization in a playful universe, Bull. Amer. Math. Soc. 77 (1971), 731-736. MR 44 \#2609.

[My] J. Mycielski, On the axiom of determinateness, Fund. Math. 53 (1964), 205-224. MR 28 \#4991.

[My-S] J. Mycielski and S. Swierczkowski, On the Lebesgue measurability and the axiom of determinateness, Fund. Math. 54 (1964), 67-71. MR 28 \#4992.

[0] J. C. Oxtoby, Measure and category, Springer, New York, 1971.

[R] H. Rogers, Jr., Theory of recursive functions and effective computability, McGraw-Hill, New York, 1967. MR 37 \#61.

[Sh] J. R. Shoenfield, Mathematical logic, Addison-Wesley, Reading, Mass., 1967. MR 37 \# 1224.

[St] J. Stern, Some measure-theoretic results in effective descriptive set theory, Israel J. Math. 20 (1975), 97-110.

Department of Mathematics, California Institute of Technology, Pasadena, CaliforNIA 91125 